\title{
Cellular magnetic resonance imaging: in vivo tracking of gastric cancer cells and detecting of lymph node metastases using microparticles of iron oxide in mice
}

This article was published in the following Dove Press journal:

Cancer Management and Research

\author{
Jian Chen ${ }^{1,2, *}$ \\ Gang Ren' \\ Rong $\mathrm{Cai}^{3}$ \\ Xiangru $\mathrm{Wu}^{4, *}$ \\ Ting Gui' \\ Jianxi Zhao' \\ Huali $\mathrm{Li}^{\prime}$ \\ Chen Guo' \\ 'Department of Radiology, Xinhua \\ Hospital, Shanghai Jiao Tong University \\ School of Medicine, Shanghai 200092, \\ People's Republic of China; ${ }^{2}$ Department \\ of Radiology, Children's Hospital of Fudan \\ University, Shanghai 201 102, People's \\ Republic of China; ${ }^{3}$ Department of \\ Radiotherapy, Ruijin Hospital, Shanghai \\ Jiao Tong University School of Medicine, \\ Shanghai 200025, People's Republic of \\ China; ${ }^{4}$ Department of Pathology, Xinhua \\ Hospital, Shanghai Jiao Tong University \\ School of Medicine, Shanghai 200092, \\ People's Republic of China
}

*These authors contributed equally to this work

Correspondence: Gang Ren Department of Radiology, Xinhua Hospital, Shanghai Jiao Tong University School of Medicine, Shanghai 200092,

People's Republic of China

Tel +86 | 365 |62 |37|

Email rengang@xinhuamed.com.cn

Rong Cai

Department of Radiotherapy, Ruijin

Hospital, Shanghai Jiao Tong University

School of Medicine, Shanghai 200025,

People's Republic of China

Tel +86 I 3788945598

Email cairong6I9@aliyun.com
Background: Monitoring the fate of implanted cells over time in an experimental animal may provide a new way to track the metastatic process. Lymph node metastase is of extremely importance for the prognostic prediction of gastric carcinoma. The aim of this study was to assess the feasibility of magnetic resonance imaging (MRI), using micron-sized superparamagnetic iron oxide particles (MPIO), for monitoring of the fate of gastric cancer cells and detecting the migration of gastric cancer cells through the lymphatic system in a mouse model.

Methods: SGC-7901 gastric cancer cells were labeled with green fluorescent MPIO. The cells were monitored in vitro at multiple time points by staining for iron-labeled cells and by flow cytometric detection of the fluorescent MPIO. MPIO-labeled cells were implanted subcutaneously into nude mice, and cellular MRI was performed at different time points until 35 days postinjection.

Results: The potential for retention of the iron particles in vitro was evaluated. Our results showed that the labeling and uptake efficiency of MPIO reached $90.0 \%$ after $24 \mathrm{hrs}$ of incubation, and a small percentage of cells that retained MPIO could be examined until 16 days after labeling. In vivo MRI-based tracking over several weeks in mice revealed regions of signal loss in the primary tumors for up to 5 weeks. Furthermore, small regions of signal void were detected in images of the inguinal lymph nodes in three mice at day 28 postinjection or later, and histological assays confirmed the presence of iron-labeled cancer cells.

Conclusion: This study supports MPIO-based cell tracking is a useful tool for monitoring the fate of gastric cancer cells in mice over time, which may facilitate progress in understanding the mechanisms of early regional lymph node micrometastases.

Keywords: magnetic resonance imaging, magnetic iron oxide particles, lymph node metastasis, gastric cancer

\section{Introduction}

Gastric cancer is a leading cause of cancer-related deaths worldwide and is the second most common invasive cancer in China. ${ }^{1,2}$ Lymph node metastasis is a major factor that affects the prognosis of gastric cancer and, thus, a key criterion for deciding on treatment strategies. Endoscopic treatment might be a consideration in patients with EGC with negligible risk of lymph node metastasis. ${ }^{3}$ Therefore, an accurate assessment of micrometastases in lymph nodes is important for early gastric cancer (EGC). 
Currently, tracking cells in vivo by magnetic resonance imaging (MRI) is common in preclinical models of disease. Cellular MRI offers the possibility to noninvasively track cells in vivo by using molecule-specific contrast agents. Superparamagnetic iron oxide nanoparticles (SPIO) are widely used for cell imaging in previous studies, however large numbers of nanoparticles must be incorporated into the targeted cells to enable their detection by MRI, and the iron-label can be diluted beyond detectability in a short time in highly-proliferative cells. ${ }^{4}$ Therefore, we try to use micron-sized superparamagnetic iron oxide particles (MPIO) to track gastric cancer cells and detect early lymph node micrometastases in this study. According to previous studies, MPIO have been successfully used to track a range of cells by MRI, ${ }^{5-7}$ while little is known about their suitability for efficient labeling of gastric cancer cells and their effects on detecting early lymph node metastases. The aim of this study was to monitor of the fate of human gastric cancer cells within primary tumors and follow the migration of these cells to the regional lymph nodes.

\section{Materials and methods}

\section{Cell culture and MPIO labeling}

The human gastric cancer cell line SGC-7901 was obtained from the Fu Dan IBS Cell Center (Shanghai, China) and maintained in RPMI-1640 (Gibco, Paisley, UK) supplemented with $10 \%$ fetal bovine serum (Gibco) and $1 \%$ penicillin-streptomycin at $37{ }^{\circ} \mathrm{C}$ and $5 \% \mathrm{CO}_{2}$ in a humidifed atmosphere. For MPIO labeling, $1 \times 10^{6}$ cells were resuspended in standard growth medium and allowed to adhere for $24 \mathrm{hrs}$. After $24 \mathrm{hrs}$, the medium was replaced with a labeling medium containing MPIO (UMC3F, Bangs Laboratories, Fishers, IN) particles, and cells were incubated for an additional $24 \mathrm{hrs}$. The MPIO beads used in this study were dragon green fluorescent polystyrene-divinylbenzene coated particle (excitation: $480 \mathrm{~nm}$, emission: $520 \mathrm{~nm}$ ) with a mean diameter of $2.85 \mu \mathrm{m}$ immersed in $9.8 \%$ magnetite $\left(\mathrm{Fe}_{3} \mathrm{O}_{4}\right)$ by weight.

\section{In vitro studies}

Varying dilutions of MPIO at doses of 12.3, 24.6, and $49.2 \mathrm{mg}$ of iron $/ \mathrm{ml}$ iron content were co-cultured within the culture medium to evaluate the uptake efficiency of SGC-7901 cells. To detect how MPIO were diluted with proliferation in vitro, the cells were harvested on days 1,4 , $7,10,13$, and 16 after cultured in the labeling medium
( $n=3$ culture dishes per time point). At each time point, cells were washed thoroughly with PBS (Gibco) to remove unincorporated MPIO from each dish, and cell viability was assessed using trypan blue exclusion. To monitor the iron concentrations in dividing cells, a cell sample was taken from each dish, centrifuged on microscopy slides, and stained for iron with Perls' Prussian blue (PPB). The number of iron-labeled cells and the total number of cells were observed in three different fields of view from each sample for each time point.

To analyze and quantify the extent of MPIO labeling, we repeated this experiment using flow cytometry based on the green fluorescence associated with MPIO. Cell samples were collected by day $1,4,7,10,13$, and 16 ( $n=3$ culture dishes per time point). The results of MPIO labeling were examined with an inverted microscope (Leica DMI3000B, Leica Microsystems) and FACS Canto II flow cytometer (BD, Biosciences). The fluorescence signal of the MPIO beads' Dragon Green label was obtained by excitation with a $480-\mathrm{nm}$ laser and detected using a 520/30 filter, and $1 \times 10^{7}$ events were acquired. At each time point, the iron content of the cells was assessed by examining the degree of fluorescence of the MPIO in each sample. Meanwhile, a sample of unlabeled SGC7901 cells was also collected as the control group.

\section{In Vivo}

\section{Animal model}

The experiments were performed in accordance with the Guidelines of the National Institutes of Health for the Care and Use of Laboratory Animals, and all the experimental protocols in this study were approved by the Ethics Committee of Xinhua Hospital Affiliated to Shanghai Jiao Tong University School of Medicine (Approval No., XHEC-F-2018-005). Twenty female BALB/c nude mice (4-5 weeks old) weighing between 18-22 g were obtained from Shanghai Experimental Animal Center (Shanghai, China) and were maintained in a specific pathogen-free environment. Unlabeled $(n=5)$ or MPIO-labeled ( $\mathrm{n}=15)$ SGC-7901 cell suspensions were injected subcutaneously into nude mice to develop solid tumors. Approximately $1 \times 10^{7}$ MPIO-labeled cells in a volume of $0.2 \mathrm{~mL}$ were injected into each animal. Animals were imaged using MRI on days 7, 14, 21, 28 and 35 after the cell injection. At each time point, three mice from the labeled group and one mouse from the control group were sacrificed for histological analysis after the imaging was completed. 


\section{MRI methods}

All mice were scanned using a clinical 3.0-Tesla MRI system (SignaHDxt, GE Medical System, Milwaukee, WI, USA) with a custom 8-channel receiver coil with $25 \mathrm{~mm}$ inner diameter (Chenguang Medical Technologies Co., Shanghai, China). Before MRI examination, all mice were anesthetized with intraperitoneal (IP) pentobarbital sodium $(50 \mathrm{mg} / \mathrm{kg}$ body weight; Sinopharm, Shanghai, China). The MR protocol consisted of following sequences, that is, 1) Transverse T2-weighted fast spin echo imaging for morphology: TR/TE, $3000 \mathrm{~ms} /$ $102.4 \mathrm{~ms}$; field of view (FOV), $40 \times 40 \mathrm{~mm}^{2}$; matrix size, 64×64; slice thickness, $1.2 \mathrm{~mm}$; and section gap, $0 \mathrm{~mm}$; and 2) T2 mapping for calculation of $\mathrm{T} 2$ value: $\mathrm{TR}$, $1000 \mathrm{~ms}$; TE $=8.25-66.0 \mathrm{~ms}$ (eight echoes, $8.25 \mathrm{~ms}$ interval between two echoes); FOV, $60 \times 60 \mathrm{~mm}^{2}$; matrix size, $192 \times 128$; slice thickness/interval, $1.2 \mathrm{~mm} / 0 \mathrm{~mm}$; number of excitations $(\mathrm{NEX})=2$.

\section{Image processing}

For quantitative analysis, all the T2 mapping images were transferred to a dedicated post-processing workstation (AW4.6, advanced workstation 4.6, GE Healthcare). The T2 mapping color images were obtained automatically with Functool software in the workstation. Blue and green represent high $\mathrm{T} 2$ values, yellow represents medium $\mathrm{T} 2$ values, and red and orange are low T2 values. Regions of interest (ROIs) were manually drawn on the center slice of each tumor on T2 mapping images, and then T2 values of the corresponding tumors were analysed quantitatively. The average $\mathrm{T} 2$ values of all these tumors were measured independently by a radiologist (Chen $\mathrm{J}$ ) who had 3 years of experience in MRI diagnosis.

\section{Histology}

The left and right inguinal lymph nodes and the primary tumors were harvested from the mice at necropsy and fixed in $10 \%$ buffered formalin overnight. Subsequently, tissue was dehydrated in $70 \%$ ethanol, and embedded in paraffin. Sections were then serially sliced at $4-\mu \mathrm{m}$ thickness with the same orientation as the MR imaging and were stained with hematoxylin and eosin (H\&E) to visualize tumor and node morphology and PPB to visualize iron.

\section{Statistical analysis}

Quantitative data were analyzed and expressed as the mean $\pm \mathrm{SD}$. The means were compared using one-way analysis of variance with GraphPad Prism 6.0 (GraphPad Software Inc., La Jolla, CA, USA). $P<0.05$ was considered statistically significant.

\section{Results}

\section{In vitro SGC-790I cell labeling}

SGC-7901 cells were labeled with MPIO in vitro experiment, and the labeling efficiency was assessed at days 1, 4, $7,10,13$, and 16 after labeling by detecting the percentage of green fluorescence associated with the MPIO using flow cytometry. SGC-7901 cells rapidly take up the MPIO while maintaining high viability. In addition, it was observed that the average iron content of SGC-7901 cells reached a plateau after $24 \mathrm{hrs}$ of incubation with MPIO at a concentration of $49.2 \mathrm{mg}$ iron $/ \mathrm{ml}$ (Figure 1). The preservation of cell viability was monitored with a trypan blue dye exclusion test to evaluate the labeling of cells with MPIO. Trypan blue cell counting showed no significant difference in cell viability between iron-labeled cells and unlabeled cells. The labeling efficiency (at the day 1 time point) was $90.0 \%$ at a concentration of $49.2 \mathrm{mg}$ iron/ $\mathrm{ml}$. Thus, we used this concentration for subsequent injection into mice during the in vivo study. As the cells divided, the number of cells that contained iron particles decreased. The percentage of labeled cells decreased to $72.3 \%$ at day $4,34.7 \%$ at day $7,11.0 \%$ at day $10,4.9 \%$ at day 13 , and $0.9 \%$ at day 16 .

The microscopy results detected at each time point were consistent with iron content analysis assessed by flow cytometry (Figure 2). Figure 2A shows the PPBstained cell samples detected at each time point. The results demonstrated the disappearance of intracellular iron from the rapidly dividing cells. Iron content detected by fluorescence microscopy also revealed similar results (Figure 2B). In addition, even after 16 days of cell division, the iron-labeled cells could still be detected by fluorescence microscopy as well as flow cytometry.

\section{In vivo MRI of MPIO-labeled SGC-790I cells $\mathrm{MRI}$ and histology of primary tumor}

Nude mice in labeled group were subcutaneously injected with SGC-7901 cell suspensions to develop solid tumors. At day 7, all growing tumors had low signal intensity throughout on transverse T2-weighted fast spin-echo imaging (not shown). At day 14, large and obvious areas of signal loss were observed within the developing masses on 
A

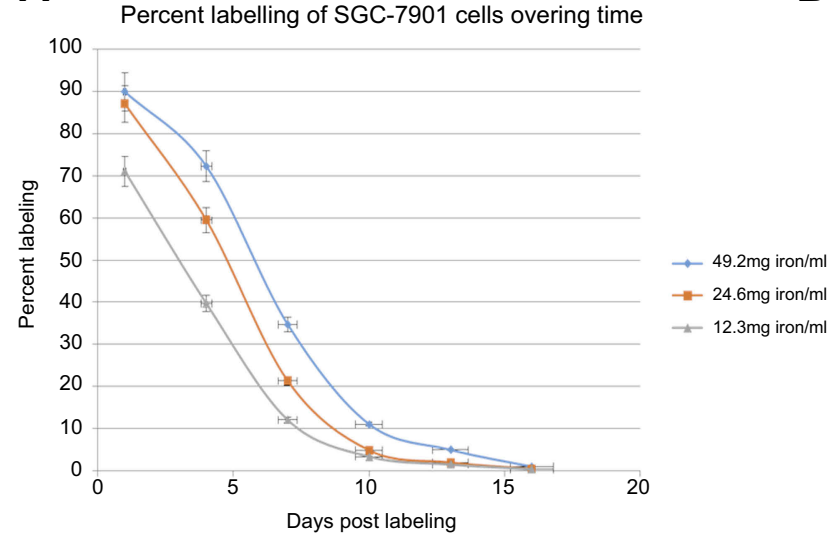

B
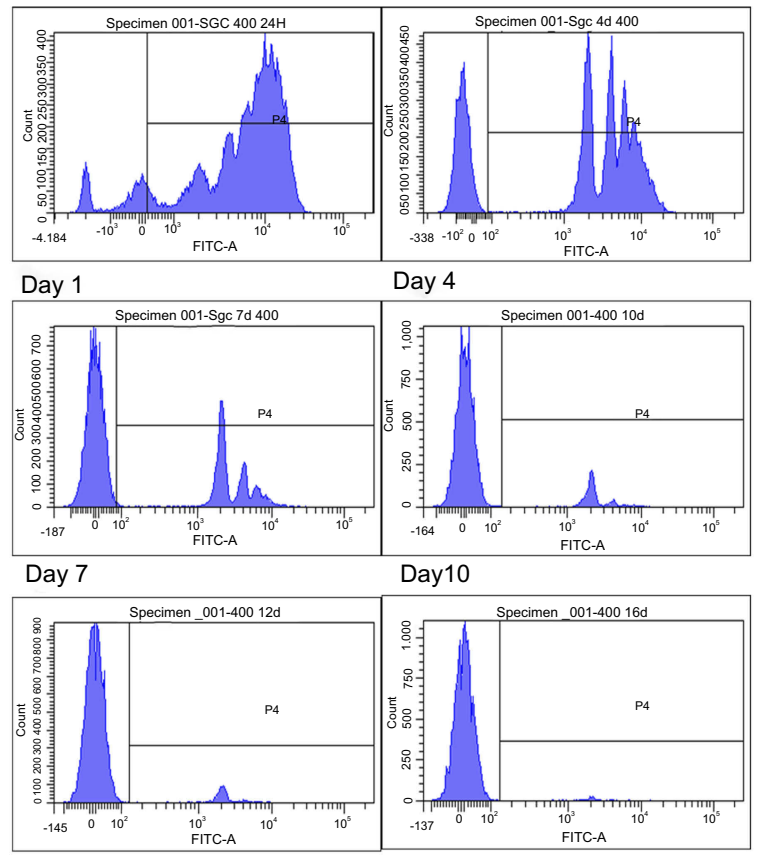

Day 13

Day 16

Figure I In vitro dilution of MPIO in SGC-790I cells that were exposed to a range of particle concentrations as measured by flow cytometry. (A) Flow cytometry data of labeling efficiency for different particle concentrations is shown. (B) Plots of flash green fluorescence intensity show that the average iron content of the SGC-790I cells reached a plateau (90.0\%) after 24 hrs of incubation with MPIO at a concentration of $49.2 \mathrm{mg}$ iron/ml (based on unlabeled control sample).

coronal T2-weighted images of the MPIO-labeled tumors (Figure 3A). In mice imaged on day 21 postinjection, continued tumor growth as well as regions of signal loss were constantly observed. T2-weighted images acquired on day 28 showed that more of the tumor mass had intermediate or high signal intensity, while the regions of low signal persisted. In mice imaged on day 35 postinjection, signal loss was still observed in all the mice in the labeled group. In addition, these areas of signal loss were located mainly in the center of all of the growing masses (Figure 3A). None of the tumors that were injected with unlabeled SGC-7901 cell suspensions showed signal loss (Figure 4A).

After MRI scanning, the tumor masses were sectioned and stained with PPB. Figure 3B shows PPB-stained sections of the primary tumors at each time point. In mice sacrificed at day 14 postinjection, a large region of pigmentation was observed. Tumors dissected at days 21, 28 and 35 after MRI scanning showed evident tumor growth as well as variable pigmentation. These results demonstrate the presence of iron-labeled cells within the tumors that were observed in the previous MRI scans.

Figure 4 shows the T2 mappings together with the MRI scans to highlight the signal intensities observed. The result showed a significant difference in $\mathrm{T} 2$ values in tumors labeled after $14,21,28$ and 35 days $(P<0.001$,
Figure 4C). In addition, mice in the experimental group had significantly lower $\mathrm{T} 2$ values compared with those in the control group $(P<0.001)$.

For iron-labeled tumors, the color of the tumors imaged on day 14 postinjection was red or orange. In addition, the average T2 value was $54.43 \mathrm{~ms}$. At day 21, some regions of the tumor were blue, while the T2WI low signal regions were marked red on the T2 map. In addition, the average T2 value increased to $68.70 \mathrm{~ms}$. The T2 mappings acquired on day 28 were yellow and blue with an average T2 value of $85.48 \mathrm{~ms}$. At day 35, as the tumors became larger, the color of tumors was green on most image slices, but the regions of signal loss on T2WI were still yellow. The average $\mathrm{T} 2$ value of the images was $67.63 \mathrm{~ms}$, probably since some of the tumors contained hemorrhage. In comparison, the color of unlabeled tumors from mice at days 14, 21, 28, and 35 postinjection was green or blue with an average $\mathrm{T} 2$ value of $74.83 \mathrm{~ms}, 72.05 \mathrm{~ms}, 66.50 \mathrm{~ms}, 113.70 \mathrm{~ms}$. Although there was heterogeneous signal intensity in some regions, the $\mathrm{T} 2$ values for unlabeled tumors were significantly increased compared to the iron-labeled tumors $(P<0.001)$.

\section{$M R I$ and histology of peripheral lymph nodes}

In mice imaged at day 28 postinjection, signal loss was observed in the bilateral inguinal lymph nodes in two of 

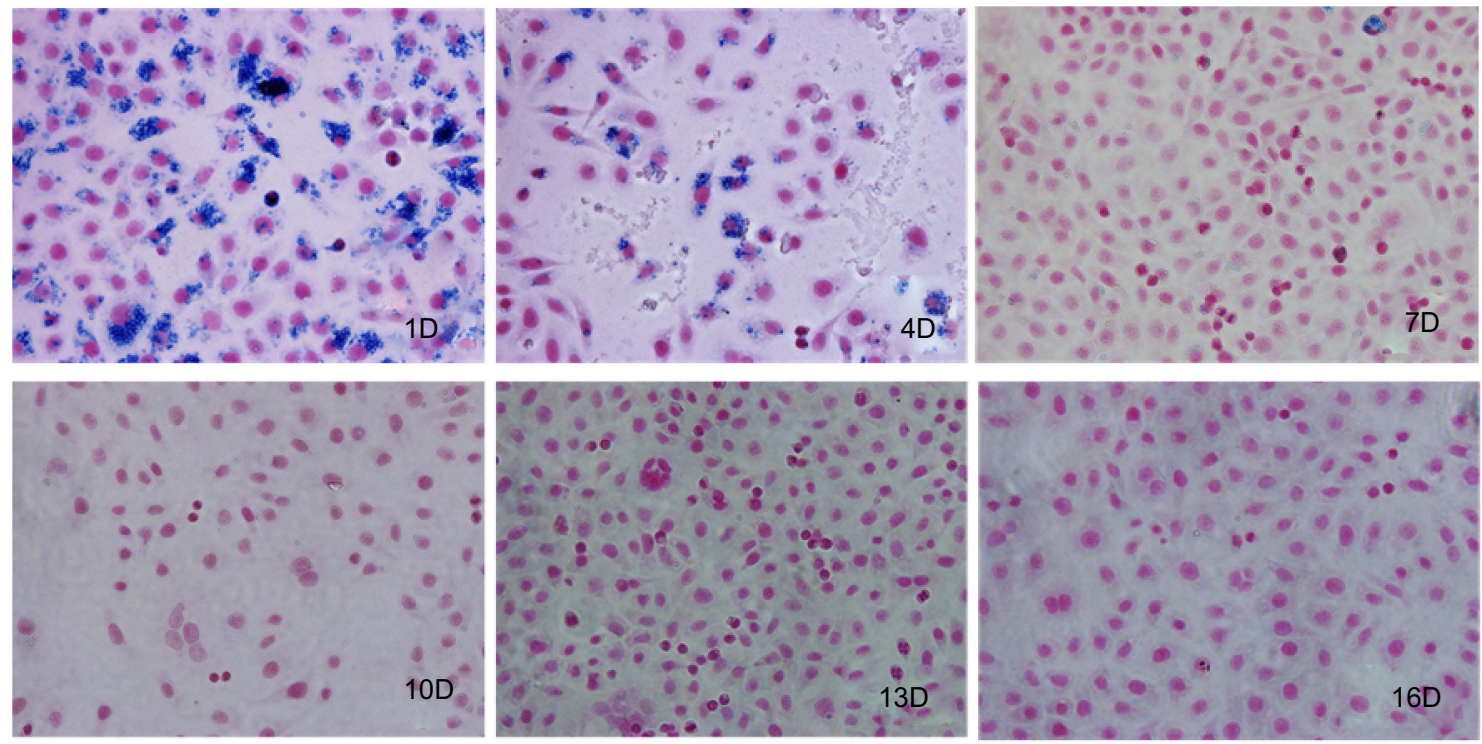

A
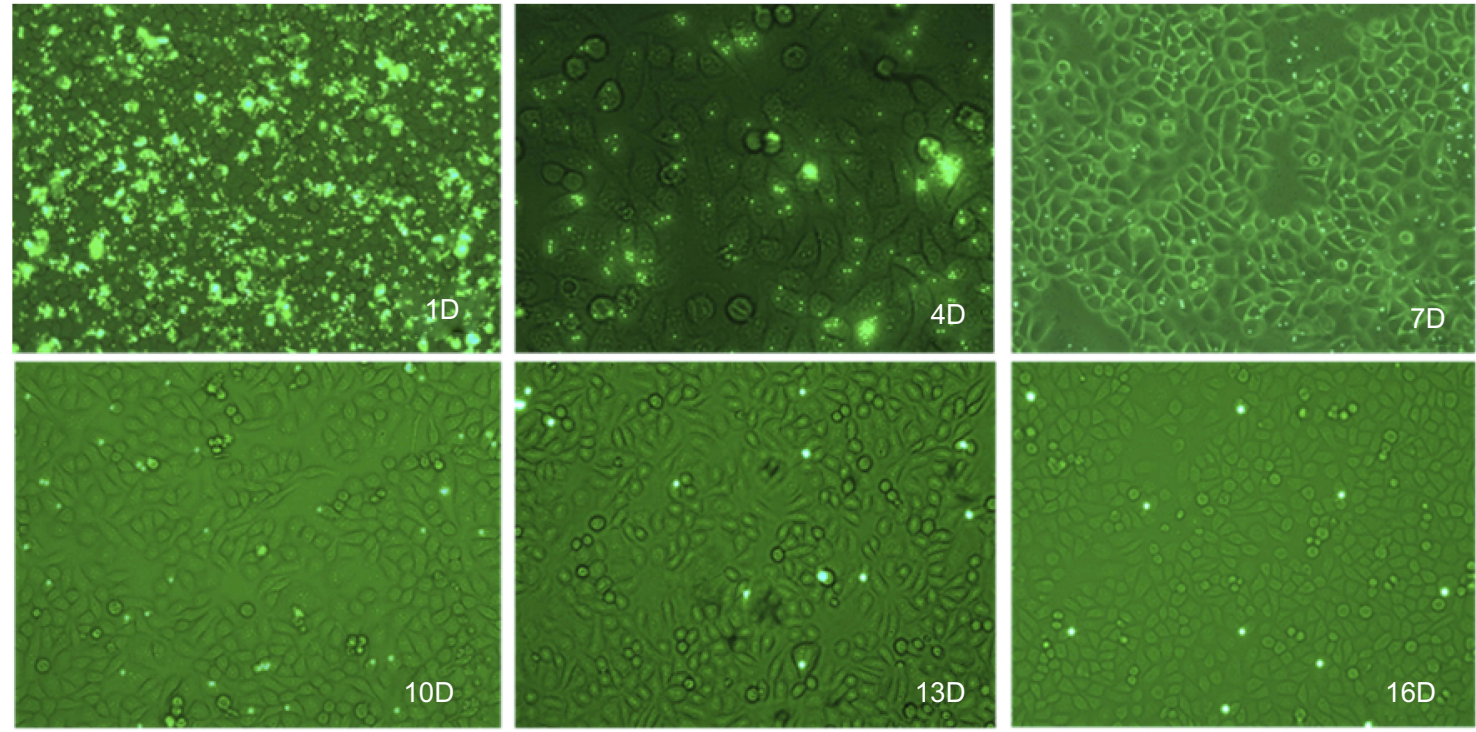

B

Figure 2 Representative cell samples of MPIO-labeled SGC-790I cells at I, 4, 7, 10, 13 and 16 days after labeling. (A) Photomicrographs of MPIO-labeled cells show the rapid disappearance of iron in dividing cells. (B) Fluorescence microscopy of MPIO-labeled SGC-790I cells show that iron-labeled cells could still be detected after 16 days of cell division.

the three mice. In mice imaged after 35 days of injection, signal loss was visible in the ipsilateral inguinal lymph node in one of the three mice. In addition, the PPB staining showed the existence of iron-positive cells in the same location as the nodes. In these mice, most of the nodes in the labeled group were obviously larger than the normal nodes, while some of the nodes appeared normal in size. There was no signal loss were observed in the lymph nodes of unlabeled mice.
In Figure 5, it was found to contain SGC-7901 cancer cells in an ipsilateral inguinal node that showed a region of signal loss on MRI at day 35. Figure 5A shows a coronal T2 weight image of a mouse at 35 days of injection. There was a heterogeneous signal loss within the primary tumor. In Figure 5B, signal loss is detected in the inguinal node by MRI at day 35. PPB staining confirmed the presence of iron-labeled cancer cells in the node (Figure 5C and D). The MRI and histology showed good correspondence. 

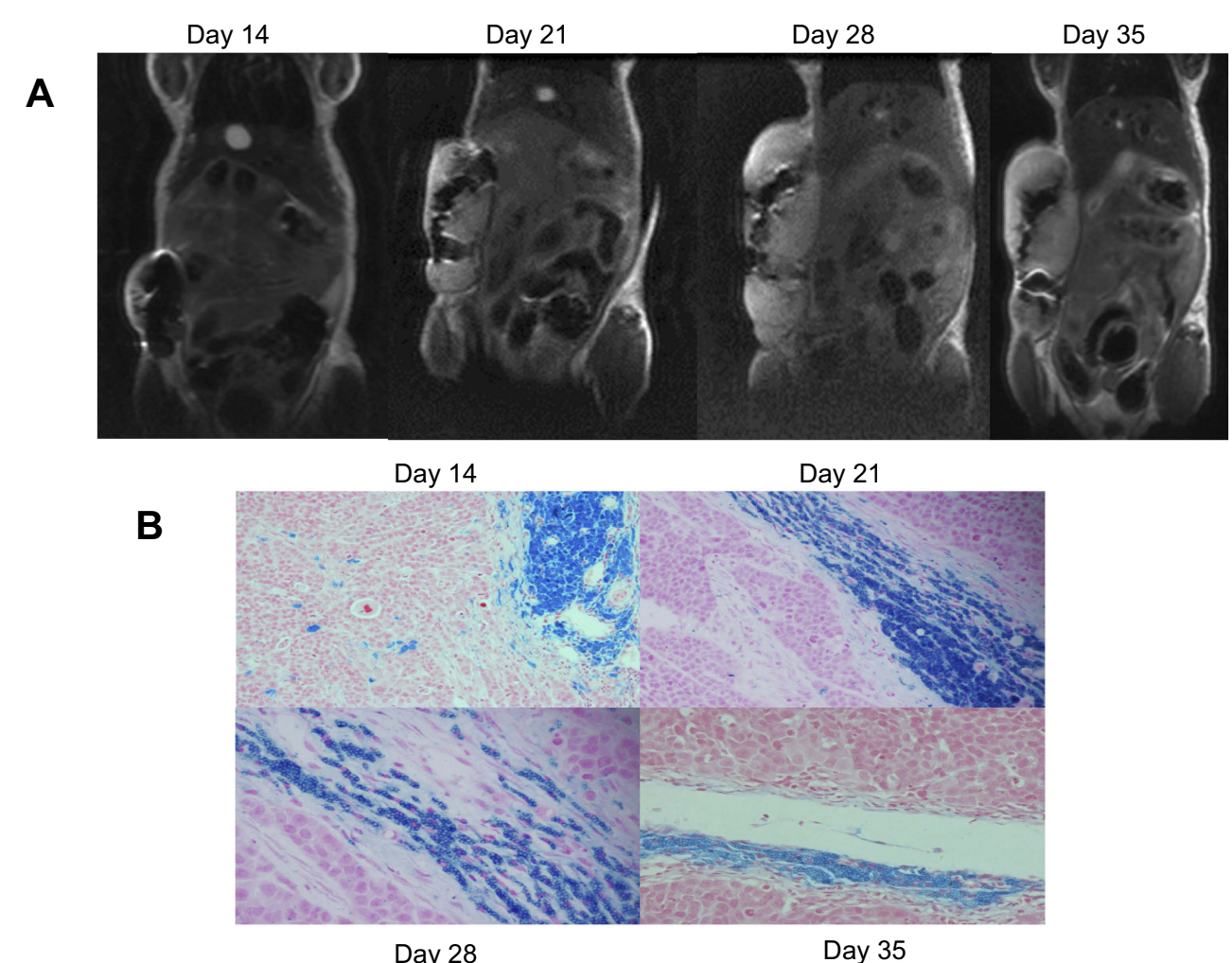

Figure 3 Coronal in vivo T2-weight images of labeled tumors over time and the corresponding PPB-stained sections. The tumor growth from iron-labeled SGC-790I cells was monitored postinjection. The signal loss caused by the iron-labeled cells was evident at day 14 postinjection and persisted as the tumor developed. PPB-stained sections show the presence of iron-labeled cells within the tumor. (A) MR image of the labeled tumor. (B) PPB-stained section showing the iron content of labeled tumor over time.

\section{Discussion}

MRI-based tracking is a hotly topic in recent years as a means of better understanding a range of diseases and potentially providing an early diagnosis. Most cellular MRI strategies in clinical trials are based on labeling with SPIO and ultrasmall (U)SPIO. Compared with USPIO and SPIO, MPIO provide a higher payload of iron oxide, which makes them an effective contrast agent for magnetic resonance imaging. A previous study reported that the number of cells labeled with MPIO was approximately threefold greater than those obtained with the best cell labeling strategies using nanometer-sized particles. ${ }^{7}$ Furthermore, it has been demonstrated that single MPIO can be detected by MRI in vitro and in mouse embryos. ${ }^{8}$ Our in vitro studies in dividing SGC-7901 cells achieved a higher labeling efficiency $(90.0 \%)$, and iron could be detected for up to 16 days. Consistent with our study, in a study about breast cancer, Economopoulo et al reported that MPIO-labeled cells could still be detected after 14 days of cell division in vitro. ${ }^{6}$ Accordingly, micron-sized particles generate a greater contrast effect when imaged by in vivo MRI and provide sensitivity to detect low levels of target.
MPIO have been widely used in experimental animal models of a range of important diseases, including multiple sclerosis, ${ }^{9}$ atherosclerosis, ${ }^{10-12}$ inflammation and cerebrovascular disorders. ${ }^{5,13}$ The use of MPIO as MRI agents allows early tracing of disease activity. Previous studies have rarely applied magnetic nanoparticles and cellular MRI to cell tracking of cancer cells since nanoparticles are progressively reduced with each cell division. Thus, it is difficult to use SPIO or USPIO to follow highly proliferating cells over long time periods. MPIO particles possess characteristics including high relaxivity, unappreciable sedimentation, and an inert polymer coat, all of which allow long-term studies of labeled cells and their progeny. A previous study showed that the infiltration of MPIOlabeled recipient immune cells could be detected by in vivo MRI for 100 days. ${ }^{14}$ These characteristics of MPIO confer more advantages over SPIO or USPIO used for labeling cancer cells. In this study, signal loss owing to MPIO-labeled cells persisted for as long as 5 weeks in developing gastric tumors. This was much longer than we observed in vitro. By contrast, a study by Foster et al reported that the dilution of SPIO in fast-growing mouse melanoma (B16F10) cancer cells to below MRI detectable 


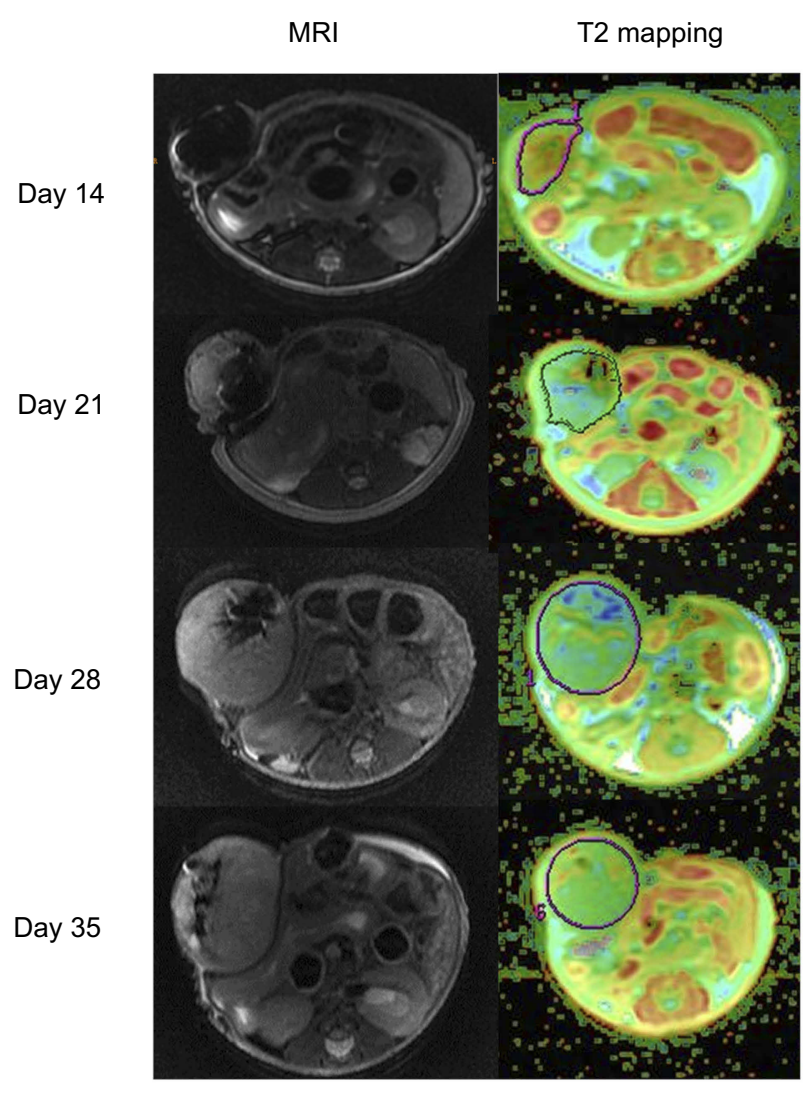

A
Labeled

\begin{abstract}
MRI
T2 mapping
\end{abstract}

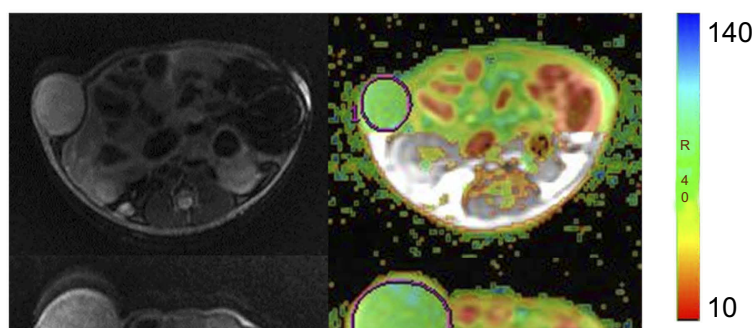

10

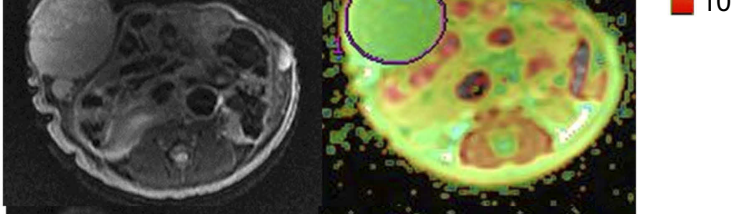

B

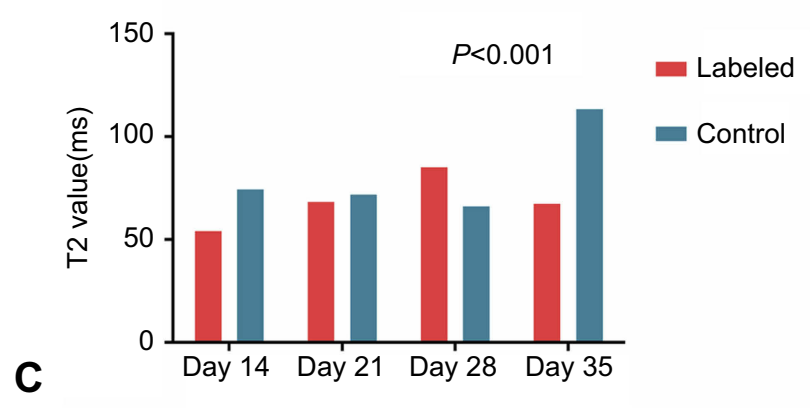

Figure 4 Appearance of labeled and unlabeled tumors over time and the corresponding T2 mapping colour images. (A, B) Conventional T2 images and pseudocolor images derived from T2 mapping images from mice at days 14, 21, 28, and 35 postinjection with labeled cells compared with images from the corresponding control groups. (C) The T2 values were compared over time between the labeled group and control group after injection of tumor cells $(P<0.00 \mathrm{I})$.

levels occurred within only 4 to 5 days after cell labeling. ${ }^{15}$ This characteristic of MPIO, which makes it more capable than other iron oxide nanoparticles, may enable investigators to detect and track cancer cells for various applications, such as detection of micrometastases from the primary tumor or monitoring response to radiotherapy and chemotherapy. Consistent with a previous study, ${ }^{6}$ our study showed that the MPIO-labeled cells made up less of the tumor mass and remained located together in one region of the tumor as the tumor became larger. According to their theory, the zone of iron-retaining cells might represent nonproliferative, or slowly cycling, cancer cells in these tumors, some of which may contribute to tumor recurrence or form late-developing metastases.

Lymph node metastasis is one of the strongest predictor of survival for patients with early gastric cancer. For patients with EGC without lymph node metastasis, according to the Japanese guidelines, endoscopic resection or more limited lymphadenectomy, such as D1 or D1+, might be options. ${ }^{16}$ However, various methods of preoperative diagnosis of 


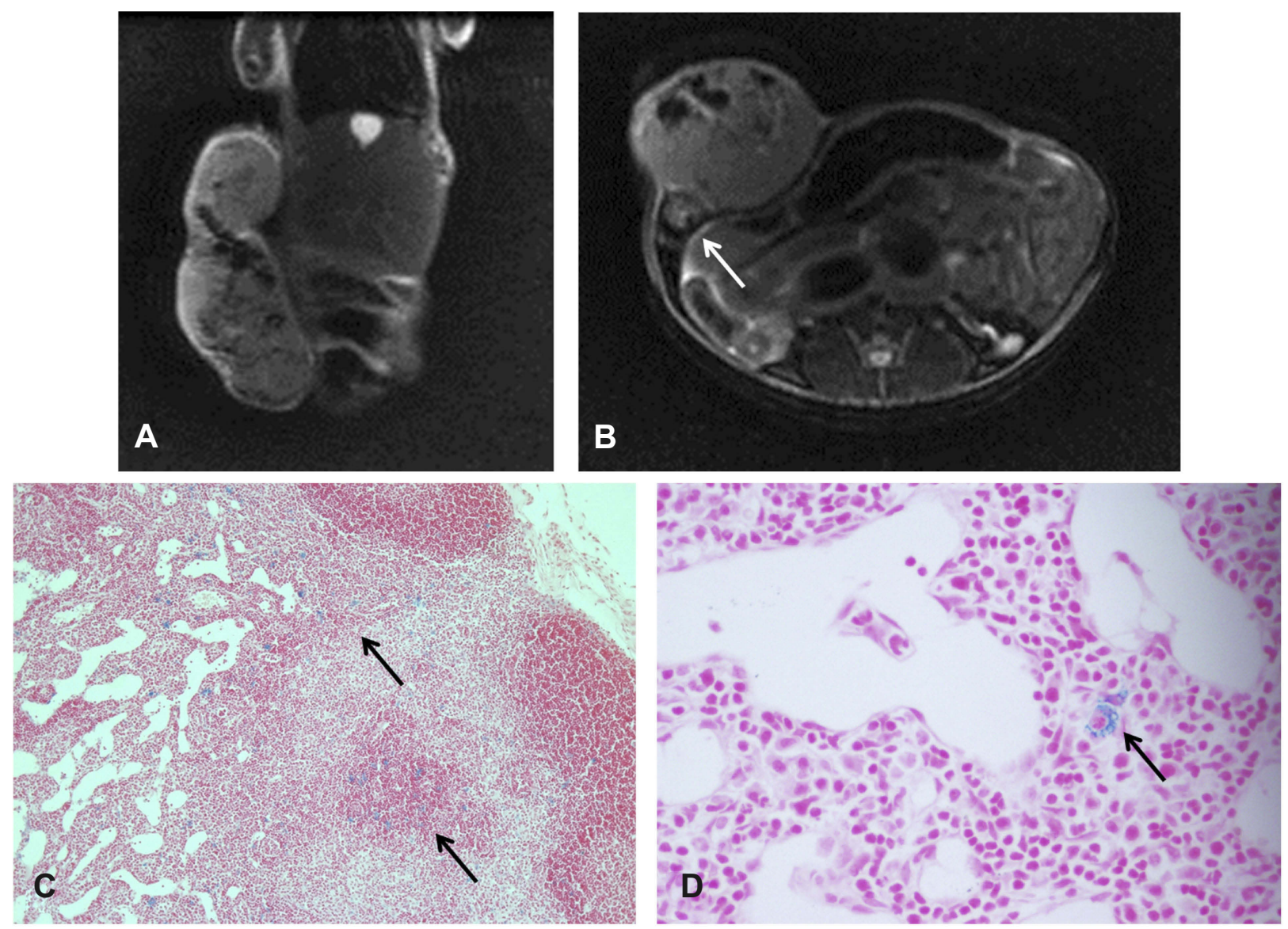

Figure $5 \mathrm{MRI}$ and iron staining of an inguinal lymph node 35 days after injection of iron-labeled SGC-790I cells in nude mice. (A) Coronal T2-weighted images of the primary tumor. (B) Axial T2-weighted image of the same mouse reveals a region of signal loss in the ipsilateral inguinal node (arrow). (C) Section of lymph node stained with PPB for iron detection at $\times 10$ magnification shows iron-positive cells in the area of the node corresponding to the region signal loss in the MR image (arrow). (D) Ironpositive area of the node at $\times 40$ magnification, where the MPIO beads can be seen (arrow), suggesting that the SGC-790I cells have retained iron particles after migrating to the node.

gastric cancer, such as computed tomography (CT) scans, endoscopic ultrasonography (EUS), and positron emission tomography (PET), have limitations for $\mathrm{N}$ staging. ${ }^{17-21} \mathrm{~A}$ meta-analysis by Luo et al showed that multidetector computed tomography (MDCT) was insufficient for detecting non-serosa-invasive GC (particularly for EGC) because of its low sensitivity. ${ }^{22}$ Meanwhile, the criteria for diagnosing metastatic nodes on $\mathrm{CT}$ imaging is still controversial. The diagnostic yield of EUS is similar to MDCT with regard to preoperative $\mathrm{N}$ staging of gastric adenocarcinoma. ${ }^{21}$ The diagnostic accuracy of standard MRI is also poor, ${ }^{17}$ it is strictly confined to the detection of lymph nodes that are enlarged or irregular. Iron oxide nanoparticles have been used to detect lymph node metastases in preclinical and clinical trial for years, and studies demonstrated that USPIO useful in the diagnosis of regional lymph node metastases in gastric cancer. ${ }^{23}$ According to the results of MRI monitoring and histopathological examination, our study demonstrated that the migration of iron-labeled gastric cancer cells from primary tumors to the peripheral lymph nodes could be detected by MRI in vivo. In mice imaged at day 28 and day 35 postinjection, signal loss on T2 weighted images that revealed the existence of iron-labeled cells in the lymph nodes was confirmed with PPB staining. This finding provides an improved understanding of the lymphatic spread of cancer and micrometastasis to lymph nodes in gastric cancer. And our study used MIPO as contrast agent will offer a new idea of earlier diagnosis of lymph node metastases in gastric cancer.

In the early stages of the experiment, we demonstrated that MPIO were readily endocytosed by gastric cancer cells and achieved a labelling efficiency of up to $90 \%$ after a relatively short co-incubation time of $24 \mathrm{~h}$. The proliferation and metabolic activity of the labeled cells were similar to those of the unlabeled cells, demonstrating that MPIO labeling was nontoxic to these cells. Similar 
results have been obtained in previous studies. ${ }^{15,24,25}$ Work in animal models indicates that MPIO have favorable blood clearance characteristics; ${ }^{26}$ however, the inert polymer coat of the MPIO used in our experimental study might be most likely accumulate and persist in the reticuloendothelial system (RES) of cells within liver and spleen, thus precluding their clinical use. ${ }^{9,26}$ As PerezBalderas et al have reported, the monomeric MPIO unsuitable for use in man because of rapid sedimentation, slow degradation and mechanical retention in organs. ${ }^{27}$ Fortunately, their study showed that multimeric MPIO might be a promising platform for the clinical use of molecular MRI contrast agents since they are non-toxic and are rapidly cleared and degraded. Moreover, a number of biodegradable MPIO are being developed, including MPIO with a coating of poly (lactide-co-glycolide) (PLGA) or cellulose, which have met with approval by US Food and Drug Administration. ${ }^{28}$

Our study has some limitations that need to be considered. First, our study was limited by the small number of experimental animals. Despite the limited sample size, the current experiment demonstrates the applicability of MPIO as potent molecular MR contrast agents for use in studies on monitoring the fate of gastric cancer cells and detecting early lymph node metastases. Second, by adding MPIO beads directly to the media that contained the SGC-7901 gastric cancer cells in vitro, we bypassed the normal steps of intravenous administration of contrast agents. Thus, our future research will investigate how to label tumor cells in situ with target-specific MPIO. Third, since distribution of signal loss was heterogeneous on MRI, hand-drawn ROIs on one axial image of the tumor may have led to sampling bias. Therefore, for an objective analysis, the averageT2 values of each primary tumor with the maximum diameter were measured on the axial image.

\section{Conclusions}

Although the application of MPIO in gastric cancer cell tracking and early lymph node metastasis detection is still limited to the animal experimental stages, it has shown great potential. Our initial experience has shown the ability of MPIO for in vivo molecular MRI monitoring of the fate of gastric cancer cells over long periods of time, which may help future studies of the progression and metastasis of gastric cancer. Moreover, by demonstrating the signal loss within regional lymph nodes in MPIOlabeled mouse model with the use of MRI, this study may provide an improved understanding of the lymphatic spread of gastric cancer and will provide the opportunity for early detection of small numbers of metastatic cells in lymph nodes.

\section{Acknowledgments}

The authors thank all the people who helped with this study. This work was partly supported by the fund of Shanghai Jiao-tong University Medical School for Scientific Research, No. 09XJ21013; Shanghai Health Bureau Fund for Scientific Research, Nos. 2010029 and 2010057; Shanghai Science and Technology Commission Fund for Scientific Research, No. 124119a0300; and Shanghai Municipal Education Commission Fund, No.12Y2034.

\section{Author contributions}

All authors contributed to data analysis, drafting or revising the article, gave final approval of the version to be published, and agree to be accountable for all aspects of the work.

\section{Disclosure}

The authors have no conflicts of interest to declare in this work.

\section{References}

1. Siegel RL, Miller KD, Jemal A. Cancer statistics, 2015. CA Cancer J Clin. 2015;65(1):5-29. doi:10.3322/caac.21254

2. Chen W, Zheng R, Baade PD, et al. Cancer statistics in China, 2015. CA Cancer J Clin. 2016;66(2):115-132. doi:10.3322/caac.21338

3. Wang Z, Zhang X, Hu J, et al. Predictive factors for lymph node metastasis in early gastric cancer with signet ring cell histology and their impact on the surgical strategy: analysis of single institutional experience. $J$ Surg Res. 2014;191(1):130-133. doi:10.1016/j. jss.2014.03.065

4. Walczak P, Kedziorek DA, Gilad A, Barnett B, Bulte J. Applicability and limitations of MR tracking of neural stem cells with asymmetric cell division and rapid turnover: the case of the shiverer dysmyelinated mouse brain.

5. Deddens LH, van Tilborg GA, van der Toorn A, et al. MRI of ICAM-1 upregulation after stroke: the importance of choosing the appropriate target-specific particulate contrast agent. Mol Imaging Biol. 2013;15 (4):411-422. doi:10.1007/s11307-013-0617-z

6. Economopoulos V, Chen Y, McFadden C, Foster PJ. MRI detection of nonproliferative tumor cells in lymph node metastases using iron oxide particles in a mouse model of breast cancer. Transl Oncol. 2013;6 (3):347-354. doi:10.1593/tlo.13121

7. Shapiro EM, Skrtic S, Koretsky AP. Sizing it up: cellular MRI using micron-sized iron oxide particles. Magn Reson Med. 2005;53(2):329338. doi: $10.1002 / \mathrm{mrm} .20342$

8. Shapiro EM, Skrtic S, Sharer K, Hill JM, Dunbar CE, Koretsky AP. MRI detection of single particles for cellular imaging. Proc Natl Acad Sci USA. 2004;101(30):10901-10906. doi:10.1073/pnas.0403918101

9. Blezer EL, Deddens LH, Kooij G, et al. In vivo MR imaging of intercellular adhesion molecule-1 expression in an animal model of multiple sclerosis. Contrast Media Mol Imaging. 2015;10(2):111-121. doi: $10.1002 / \mathrm{cmmi} .1602$ 
10. Yan F, Yang W, Li X, et al. Magnetic resonance imaging of atherosclerosis using CD81-targeted microparticles of iron oxide in mice. Biomed Res Int. 2015;2015:758616. doi:10.1155/2015/ 758616

11. McAteer MA, Akhtar AM, von Zur Muhlen C, Choudhury RP. An approach to molecular imaging of atherosclerosis, thrombosis, and vascular inflammation using microparticles of iron oxide. Atherosclerosis. 2010;209(1):18-27. doi:10.1016/j.atherosclero sis.2009.10.009

12. von Elverfeldt D, von Zur Muhlen C, Wiens K, et al. In vivo detection of activated platelets allows characterizing rupture of atherosclerotic plaques with molecular magnetic resonance imaging in mice. PLoS One. 2012;7(9):e45008. doi:10.1371/journal.pone.0045008

13. McAteer MA, von Zur Muhlen C, Anthony DC, Sibson NR, Choudhury RP. Magnetic resonance imaging of brain inflammation using microparticles of iron oxide. Methods Mol Biol. 2011;680:103115. doi:10.1007/978-1-60761-901-7 7

14. Ye Q, Wu YL, Foley LM, et al. Longitudinal tracking of recipient macrophages in a rat chronic cardiac allograft rejection model with noninvasive magnetic resonance imaging using micrometer-sized paramagnetic iron oxide particles. Circulation. 2008;118(2):149156. doi:10.1161/CIRCULATIONAHA.107.746354

15. Foster PJ, Dunn EA, Karl KE, et al. Cellular magnetic resonance imaging: in vivo imaging of melanoma cells in lymph nodes of mice. Neoplasia. 2008;10(3):207-216. doi:10.1593/neo.07937

16. Japanese gastric cancer treatment guidelines 2010 (ver. 3). Gastric cancer: official journal of the International Gastric Cancer Association and the Japanese Gastric Cancer Association. Gastric Cancer.2011;14 (2):113-123.

17. Kwee RM, Kwee TC. Imaging in assessing lymph node status in gastric cancer. Gastric Cancer. 2009;12(1):6-22. doi:10.1007/ s10120-008-0492-5

18. Barros RH, Penachim TJ, Martins DL, Andreollo NA, Caserta NM. Multidetector computed tomography in the preoperative staging of gastric adenocarcinoma. Radiologia Bras. 2015;48(2):74-80. doi:10.1590/0100-3984.2014.0021

19. Cardoso R, Coburn N, Seevaratnam R, et al. A systematic review and meta-analysis of the utility of EUS for preoperative staging for gastric cancer. Gastric Cancer. 2012;15(Suppl 1):S19-S26. doi:10.1007/s10120-011-0115-4
20. Wu CX, Zhu ZH. Diagnosis and evaluation of gastric cancer by positron emission tomography. World J Gastroenterol. 2014;20 (16):4574-4585. doi:10.3748/wjg.v20.i16.4574

21. Cimavilla Román M, de la Serna Higuera C, Loza Vargas LA, et al. Endoscopic ultrasound versus multidetector computed tomography in preoperative gastric cancer staging. Rev Esp De Enfermedades Digestivas. 2017;109(11):761-767. doi:10.17235/reed.2017.4638/ 2016

22. Luo M, Lv Y, Guo X, Song H, Su G, Chen B. Value and impact factors of multidetector computed tomography in diagnosis of preoperative lymph node metastasis in gastric cancer: a PRISMA-compliant systematic review and meta-analysis. Medicine. 2017;96(33): e7769. doi:10.1097/MD.0000000000007769

23. Tokuhara T, Tanigawa N, Matsuki M, et al. Evaluation of lymph node metastases in gastric cancer using magnetic resonance imaging with ultrasmall superparamagnetic iron oxide (USPIO): diagnostic performance in post-contrast images using new diagnostic criteria. Gastric Cancer. 2008;11(4):194-200. doi:10.1007/s10120008-0480-9

24. Hinds KA, Hill JM, Shapiro EM, et al. Highly efficient endosomal labeling of progenitor and stem cells with large magnetic particles allows magnetic resonance imaging of single cells. Blood. 2003;102 (3):867-872. doi:10.1182/blood-2002-12-3669

25. Boulland JL, Leung DS, Thuen M, et al. Evaluation of intracellular labeling with micron-sized particles of iron oxide (MPIOs) as a general tool for in vitro and in vivo tracking of human stem and progenitor cells. Cell Transplant. 2012;21(8):1743-1759. doi:10.3727/096368911X627598

26. Jefferson A, Wijesurendra RS, McAteer MA, Choudhury RP. Development and application of endothelium-targeted microparticles for molecular magnetic resonance imaging. Wiley Interdiscip Rev. 2012;4(3):247-256. doi:10.1002/wnan.1164

27. Perez-Balderas F, van Kasteren SI, Aljabali AAA, et al. Covalent assembly of nanoparticles as a peptidase-degradable platform for molecular MRI. Nat Commun. 2017;8:14254. doi:10.1038/ ncomms 14254

28. Nkansah MK, Thakral D, Shapiro EM. Magnetic poly(lactide-coglycolide) and cellulose particles for MRI-based cell tracking. Magn Reson Med. 2011;65(6):1776-1785. doi:10.1002/ mrm.22765

\section{Publish your work in this journal}

Cancer Management and Research is an international, peer-reviewed open access journal focusing on cancer research and the optimal use of preventative and integrated treatment interventions to achieve improved outcomes, enhanced survival and quality of life for the cancer patient.
The manuscript management system is completely online and includes a very quick and fair peer-review system, which is all easy to use. Visit http://www.dovepress.com/testimonials.php to read real quotes from published authors. 